\title{
Clinical Medicine Insights: Blood Disorders
}

\section{Nonsteroidal Anti-inflammatory Drug Induced Thrombotic Thrombocytopenic Purpura}

\author{
Karlos Z. Oregel ${ }^{1}$, Jeremy Ramdial ${ }^{1}$ and Stefan Glück $^{2}$ \\ 1Department of Internal Medicine, University of Miami/Jackson Memorial Hospital, University of Miami Leonard M. Miller School of Medicine, \\ 1400 NW 10th Avenue, Suite 509b, Miami, FL 33126, USA. ²Department of Medicine, Division of Hematology/Oncology Sylvester \\ Comprehensive Cancer Center, University of Miami, Leonard M. Miller School of Medicine, Miami, Florida.
}

\begin{abstract}
A 21-year-old male presented to the emergency department after a 5-day history of recurrent vomiting and decreased urine output. History revealed ingestion of ibuprofen. During the diagnostic workup, the following was identified: white blood cell count $13.4(\times 10(3) / \mathrm{mcL})$, hemoglobin $11.9(\times 10(6) / \mathrm{mcL})$ with an MCV of $73 \mathrm{fL}$, hematocrit $34 \%$ and platelets were $31,000 / \mathrm{mcL}$, sodium of $130 \mathrm{mmol} / \mathrm{L}$, potassium of $5.1 \mathrm{mmol} / \mathrm{L}$, chloride of $83 \mathrm{mmol} / \mathrm{L}$, bicarbonate of $21 \mathrm{mmol} / \mathrm{L}$, blood urea nitrogen of $184 \mathrm{mg} / \mathrm{dL}$ and creatinine of $19.1 \mathrm{mg} / \mathrm{dL}$. He was later diagnosed with thrombotic thrombocytopenic purpura (TTP) based on the fact that he presented with most components of the TTP pentad (except for fever), which included altered mental status, acute kidney injury, thrombocytopenia, and evidence of red cell fragmentation and his ADAMTS13 level was found to be less than $10 \%$ prior to therapy. The patient then received plasma exchange, oral corticosteroids, and hemodialysis, which led to a full recovery of platelet count and renal function.
\end{abstract}

KEYWORDS: TTP, NSAIDs, Ibuprofen, drug-induced TTP, thrombotic thrombocytopenic purpura

CITATION: Oregel et al. Nonsteroidal Anti-inflammatory Drug Induced Thrombotic Thrombocytopenic Purpura. Clinical Medicine Insights: Blood Disorders 2013:6 19-22 doi:10.4137/CMBD.S12843.

TYPE: Case Report

FUNDING: Authors disclose no funding sources.

COMPETING INTERESTS: Authors disclose no potential conflicts of interest.

COPYRIGHT: () the authors, publisher and licensee Libertas Academica Limited. This is an open-access article distributed under the terms of the Creative Commons CC-BY-NC 3.0 License.

CORRESPONDENCE: s.gluck@med.miami.edu

Drug-induced TTP was described after use of the following agents that commonly include quinine, antiplatelet agents (eg, ticlopidine, clopidogrel), and chemotherapeutic agents. ${ }^{1}$ A literature search for nonsteroidal anti-inflammatory drugs as the precipitant factor produced only one case report by Catizone et al from 1974 that described the development of TTP secondary to ibuprofen. Here, we describe the only other case in the literature since then. In 1986, 100 million prescriptions were written for NSAIDs worldwide. With the increasing availability and use of NSAIDs, it is important to be aware that these medications are a potential cause of TTP. Ultimately, establishing a diagnosis with appropriate treatment is of utmost importance in preventing further complications and mortality.

Case

Here, we describe a case of a Hispanic male with thrombotic thrombocytopenic purpura induced by nonsteroidal anti-inflammatory drugs (NSAIDs). The patient was a 21-year-old Latino male who presented to the emergency department reporting a five day history of nausea, vomiting, and decreased urine output. The patient reported that symptoms began shortly after a purposeful consumption of a large quantity of ibuprofen (600-mg pills) in a suicide attempt. The exact amount of ibuprofen is unclear, but it was predicted that he consumed approximately 18 grams of ibuprofen. The past medical history was only notable for hypertension diagnosed three years prior, for which he was not receiving any medical therapy. Surgical history consisted of an appendectomy. The patient noted a prior recreational use of alcohol, marijuana and cocaine use, but he denied any use in the past few months. Family history was unremarkable for blood disorders and heart or renal disease. On initial exam, the patient had a temperature of $35.8^{\circ} \mathrm{C}$, a heart rate of 56 beats per minute, a blood pressure of $122 / 70 \mathrm{mmHg}$, a respiratory rate of 18 breaths per 
minute and his oxygen saturation was $100 \%$ on room air. The patient was noted to be alert and oriented despite appearing drowsy. On physical exam, pertinent findings included jaundice, icteric sclera, dry mucous membranes, and generalized petechiae. Heart and lung examination were unremarkable. The abdomen was found to be soft but slightly tender to palpation at the right upper quadrant. No rebound or guarding was appreciated. Hepatomegaly was appreciated on initial but not subsequent physical exams. No renal bruits were appreciated. Neurological exam was unremarkable and grossly non-focal.

Laboratory findings included chemistry panel with a sodium of $130 \mathrm{mmol} / \mathrm{L}$, potassium of $5.1 \mathrm{mmol} / \mathrm{L}$, chloride of $83 \mathrm{mmol} / \mathrm{L}$, bicarbonate of $21 \mathrm{mmol} / \mathrm{L}$, blood urea nitrogen of $184 \mathrm{md} / \mathrm{dL}$ and creatinine of $19.1 \mathrm{mg} / \mathrm{dL}$, and glucose of $95 \mathrm{mg} / \mathrm{dL}$. Serum osmolality was $323 \mathrm{mOsm} / \mathrm{kg}$, with an anion gap calculated at 26 . White blood cell count $13.44(\times 10(3) / \mathrm{mcL})$, hemoglobin was $11.9(\times 10(6) / \mathrm{mcL})$ with an $\mathrm{MCV}$ of $73 \mathrm{fL}$, hematocrit was $34 \%$, and platelets were $31,000 / \mathrm{mcL}$. Total bilirubin was $24.6 \mathrm{mg} / \mathrm{dL}$ (direct bilirubin, $20 \mathrm{mg} / \mathrm{dL}$ ), liver function tests were elevated with aspartate aminotransferase was 105 units/L, alanine aminotransferase was 167 units/L, and alkaline phosphatase was 464 units/L. International normalized ratio and prothrombin time were within normal limits. Lactate dehydrogenase (LDH) was 8747 units/L, and haptoglobin was less than $10 \mathrm{mg} / \mathrm{dL}$. On the peripheral smear, schistocytes, helmet cells, and large platelets were noted. Drug urine toxicology was negative for any illicit drugs or alcohol. The patient was also HIV-, hepatitis B-, and hepatitis C-negative. Urinalysis showed mild proteinuria, hematuria, and bilirubinemia. Acetaminophen level was undetectable. Ultrasound of the abdomen was unremarkable and hepatomegaly and atrophy of kidneys was not appreciated. Results of the various tests are shown in Table 1.

The initial diagnostic impression suggested that the patient had developed thrombotic thrombocytopenic purpura. The patient immediately underwent plasma exchange for a total duration of 5 days and simultaneously started on oral glucocorticoids (prednisone $1 \mathrm{mg} / \mathrm{kg}$ ). Following treatment, platelet count recovered and remained above 150,000 after

Table 1. CBC and BMP values compared to reference ranges used at local hospital of the patient described in the case as well as a patient described in the Catizone et al case report several years ago.

\begin{tabular}{|c|c|c|c|c|c|c|c|}
\hline & $\mathrm{Na}^{+}$ & $\mathbf{K}^{+}$ & $\mathrm{Cl}^{-}$ & $\mathrm{HCO}_{3}$ & BUN & $\mathrm{Cr}$ & Glu \\
\hline${ }^{*} \mathrm{Pt}$ & 130 & 5.1 & 83 & 21 & 184 & 19.19 & 95 \\
\hline Prev. Pt & - & 6 & - & - & 208 & $3 \mathrm{mg} \%$ & - \\
\hline${ }^{*}$ Ref. & $137-145$ & $3.6-5$ & $98-107$ & $22-30$ & $9-20$ & $.66-1.25$ & $74-106$ \\
\hline & \multicolumn{2}{|c|}{ WBC } & \multicolumn{2}{|l|}{$\mathrm{Hb}$} & Het & \multicolumn{2}{|c|}{ PIt } \\
\hline${ }^{*} \mathrm{Pt}$ & \multicolumn{2}{|c|}{13.4} & \multicolumn{2}{|l|}{11.9} & 34.6 & \multicolumn{2}{|c|}{31} \\
\hline Prev. Pt & \multicolumn{2}{|l|}{ - } & \multicolumn{2}{|l|}{14.3} & - & \multicolumn{2}{|c|}{60} \\
\hline${ }^{*}$ Ref. & \multicolumn{2}{|c|}{$4.3-11$} & \multicolumn{2}{|l|}{$14-18$} & $42-52$ & \multicolumn{2}{|r|}{$140-440$} \\
\hline
\end{tabular}

2 days. Concomitantly, the patient underwent aggressive rehydration with intravenous fluids but remained anuric. The patient was then started on renal replacement therapy. Following the initiation of intermittent hemodialysis, creatinine and blood urea nitrogen improved significantly. Additionally, the $\mathrm{LDH}$ returned to baseline. An initial transaminitis on presentation subsequently resolved. Psychiatry also evaluated the patient and concluded that the suicidal incident was rooted to an adjustment disorder. The patient was then discharged after an uneventful hospital course. A few weeks later, the patient was seen in the outpatient setting where he continued to report resolution of all symptoms and normal urination. Prednisone and renal replacement therapy were discontinued at that point.

\section{Discussion}

Thrombotic thrombocytic purpura is a rare blood disorder characterized by clotting of small blood vessels and low blood platelet counts. A pentad often characterizes TTP that includes neurological deficits (often in the form of altered mental status), thrombocytopenia, fever, renal dysfunction, and evidence of microangiopathic hemolytic anemia. Not all features must be present for a diagnosis, but important primary criteria include thrombocytopenia and microangiopathy. ${ }^{3}$ The diagnosis of TTP is made after an exclusion of more prevalent multisystem diseases such as disseminated intravascular coagulation, sepsis, malignant hypertension, systemic lupus erythematosus, and hematologic malignancies. ${ }^{2}$

TTP is a rare disease with approximately 3.7 cases per million per year in the United States. In approximately $90 \%$ of patients, the cause of TTP is unknown. ${ }^{4,5}$ Known conditions that are associated with TTP include a postpartum state, postinfection (Escherichia coli 0157:H7, E. coli 0111, E. coli 0104:H4), pancreatitis, malignancy, post-surgical, HIV, postpneumococcal infection, and drug-induced.

For the association with drugs, quinine is the most commonly identified. ${ }^{6}$ Other documented drugs include oral contraceptives, extended-release opioids, valacyclovir and chemotherapeutic agents such as mitomycin $\mathrm{C}$, gemcitabine, cisplatin, oxaliplatin, pentostatin, bevacizumab, and sunitinib. Additionally, the chemotherapeutic agents are typically dose-dependent in their involvement with TTP, and therapies such plasma exchange have been found to be ineffective. ${ }^{4,6}$ Other widely used medications such as the anti-platelet agents Ticlopidine and clopidogrel have also been associated with development of TTP. The underlying mechanisms of how these drugs cause TTP largely remains unknown. It is not clear if there is a direct effect of the drug or its metabolites on the vasculature or if a secondary immune response is responsible. ${ }^{3}$ However, the underlying mechanism for how platelet consumption occurs and the thrombotic microangiopathy that ensues is well-understood.

TTP is associated with a deficiency of a protein known as ADAMTS13 (a disintegrin and metalloprotease with a 
thrombospondin type 1 motif, member 13). The deficiency can be acquired, or, less likely, congenital. The gene for the ADAMTS13 protein is located on chromosome 9q34. For the congenital form, several mutations have been identified, leading to the deficiency. This protein functions to cleave a large von Willebrand factor (UL-VWf) multimer (derived from endothelial cells) into a smaller form. ${ }^{7}$ If the UL VWfmultimer cannot be cleaved, it remains in circulation and activated platelets adhere to it. Platelets are then consumed in the process and the microvascular is further injured from the high shear stress. ${ }^{7}$ The platelet-UF VWF complexes, if large enough, can also thrombose in the microvasculature of multiple organs. Clinically, the ADAMTS13 protein level is a useful tool in reaching the diagnosis of TTP. It is important to recognize that levels of this protein can be variable and even normal in several of the drugs associated with TTP. ${ }^{8}$ In one study, the variability in TTP ranged from $13-70 \% .{ }^{9}$ Therefore, a decrease in ADAMTS13 activity and the presence of its antibody is more complementary to the diagnosis and overall clinical picture. A decrease in activity can be associated with TTP if less than 5-10\% (detection range 0.5-100\%). The overall consensus is that the presence of an ADAMTS13 deficiency is a critical underlying risk factor, but requires a secondary trigger to develop TTP. ${ }^{4}$ In clinical practice, if a diagnosis is even suspected, it is imperative to consider initiating plasma exchange (plasmapheresis with infusions of fresh frozen plasma) immediately. ${ }^{3}$ Plasma exchange therapy for acquired TTP is effective because it removes ADAMTS13 autoantibodies and UL-VWF multimers, as well as prevents further supply of ADAMTS13. ${ }^{7}$ Disease activity can then be tracked by monitoring LDH and platelets. Notably, prior to the availability of plasma exchange, patient survival was about $10 \%$. With the advent of plasma exchange technology, the overall response rate is now estimated at $80-90 \%{ }^{3,4}$ The therapy options include plasma exchange, and recent studies have also shown a benefit in using rituximab as a first line therapy of acute acquired TTP. ${ }^{7,10}$ The rituximab was used in conjunction with plasma exchange and corticosteroids and administered at $375 \mathrm{mg} / \mathrm{m}^{2}$ intravenously within the first three days of admission and diagnosis with shown clinical benefit. ${ }^{10}$

The chemical compound 2-(4-isobutylphenyl) propionic acid, also known as ibuprofen, is a nonsteroidal anti-inflammatory agent used in the management of pain, inflammation, fever, etc. In the the US alone about 30 billion doses are utilized each year. ${ }^{11}$ The drug inhibits both cyclooxygenase 1 and 2 that prevents the production of thromboxanes and prostaglandins upon inhibition induce an anti-inflammatory, analgesic, and antipyretic effect. It is well characterized that the inhibition of the COX-1 pathway is responsible for most of the unwanted side effects. ${ }^{12}$ These commonly include nausea, dyspepsia, gastrointestinal bleeding, transaminitis, and, less likely, jaundice, fatal fulminant hepatitis, liver necrosis, and renal papillary necrosis and occasionally hepatic failure. Ibuprofen is plasma protein bound with a large volume of distribution $(0.11-0.19 \mathrm{~L} / \mathrm{kg})$. A single $400-\mathrm{mg}$ dose in adults produces a peak plasma level at 1-2 hours after ingestion and is eliminated via the kidneys within the next $8 \mathrm{~h}$ and then is practically undetectable by $12 \mathrm{~h}$. Its maximum recommended daily dose is $1200-3200 \mathrm{mg} \cdot{ }^{12-14}$ Anemia is the only known hematological effect. ${ }^{15}$ More serious effects associated with long-term use and can manifest as esophageal ulceration and chronic renal failure and can worsen heart failure. ${ }^{16}$ NSAID overdose is generally a benign process. In 2010, the Annual Report of the American Association of Poison Control Centers National Poison Data System (NPDS): 28th Annual Report reported 65,699 cases of overdose with 9,169 of those characterized as intentional. Forty-seven cases were classified as a serious toxicity that manifested as status epilepticus, respiratory failure, ventricular arrhythmias, or cardiac arrest. No deaths due to ibuprofen were reported in 2010. The high index of safety centers around ibuprofen's inability to accumulate in the body regardless of subsequent doses. ${ }^{17}$

There have been no recent cases attributing the development of TTP to patients exposed to NSAIDS. One case report from 1974 described a 55-year-old Italian female who developed TTP after ingesting $900 \mathrm{mg}$ of ibuprofen. No other alternative etiologies could be accounted for and the patient eventually expired as no plasma exchange therapy was available at that time. ${ }^{2}$ However, there have been some reports of NSAIDS causing hemolytic uremic syndrome (HUS), a disease process very similar to TTP. ${ }^{18}$ Paradoxically, antiplatelet therapy was at one point considered as potential treatment for TTP. The theory stipulated that by preventing platelet aggregation with antiplatelet agents, further endothelial injury and thrombosis could be prevented. Data from this study demonstrated that at least in certain cases, antiplatelet drugs probably play only a limited role in the treatment of patients with TTP. ${ }^{19}$ In another trial, prostacyclin (PGI2) infusion, again with the same principle, failed to reverse platelet aggregation caused by TTP. ${ }^{20}$

\section{Conclusion}

Our patient presented with clinical symptoms that included altered mental status, acute renal dysfunction, thrombocytopenia, and evidence of red blood cell fragmentation. All symptoms evolved a few hours after ingesting a large amount of ibuprofen. In our assessment, other possible disease entities were considered but excluded such as hemolytic uremic syndrome (no history of diarrhea and negative E. coli 0157H7 study), autoimmune hemolytic anemia (Coombs test was negative), disseminated intravascular coagulation (coagulation factors were normal), HIV, and absence of other known drugs. ADAMTS13 activity was less than $10 \%$ and solidified our assessment that TTP was the clear diagnosis. Most importantly, clinically, the patient showed full recovery following successful plasma exchange. The mechanism of how NSAIDs could have precipitate TTP remains to be further investigated. Ibuprofen, its metabolites or even components of the 
drug formula may have caused this condition. Nonetheless, because of the widespread use of NSAIDs and the high mortality associated with undiagnosed TTP, we present this case-report to shed light into a possible, unknown association between ibuprofen and TTP.

\section{Author Contributions}

Wrote the first draft of the manuscript: KO. Contributed to the writing of the manuscript: JR, SG. Agree with manuscript results and conclusions: KO, JR, SG. Jointly developed the structure and arguments for the paper: KO, JR, SG. Made critical revisions and approved final version: KO, JR, SG. All authors reviewed and approved of the final manuscript.

\section{DISCLOSURES AND ETHICS}

As a requirement of publication the authors have provided signed confirmation of their compliance with ethical and legal obligations including but not limited to compliance with ICMJE authorship and competing interests guidelines, that the article is neither under consideration for publication nor published elsewhere, of their compliance with legal and ethical guidelines concerning human and animal research participants (if applicable), and that permission has been obtained for reproduction of any copyrighted material. This article was subject to blind, independent, expert peer review. The reviewers reported no competing interests.

\section{REFERENCES}

1. George JN. The TiborGreenwalt Award Lecture. The Oklahoma Thrombotic Thrombocytopenic Purpura-Hemolytic Uremic Syndrome Registry: a program for patient care, education and research. Transfusion. 2004;44(9):1384-1392.

2. Catizone L, Santoro A, Scialfa G, Cagnoli L, Fabbri L. [Thrombotic thrombocytopenic purpura due to administration of Ibuprofen]. Minerva Nefrol. 1974;21(6):439-444. In Italian.

3. Steensma DP, Pruthi RK. In: Ghosh AK, ed. Mayo Clinic Internal Medicine Review: Thrombotic thrombocytopenic Purpura. 8th ed. Rochester, MN: Scientific Publications. 2008:422-423.

4. George JN. Evaluation and management of patients with thrombotic thrombocytopenic purpura. Journal of Intensive Care Medicine. 2007;22(2):82-91.
5. Scully M, Hunt BJ, Benjamin S, et al; British Committee for Standards in Haematology. Guidelines on the diagnosis and management of thrombotic thrombocytopenic purpura and other thrombotic microangiopathies. Br J Haematol. 2012;158(3):323-335.

6. George JN. How I treat patients with thrombotic thrombocytopenic purpurahemolytic uremic syndrome. Blood. 2000;96(4):1223-1229.

7. Matsumoto M. anti-ADAMTS13 autoantibodies in patients with thrombotic thrombocytopenic purpura. Nihon Rinsho Meneki Gakkai Kaishi. 2013; 36(2):95-103.

8. Boctor FN, Smith JA. Diagnosis of thrombotic thrombocytopenic purpura with normal ADAMTS13 activity and absence of its inhibitor (anti-ADAMTS13 antibodies). American Journal of Hematology. 2008;83(3):256.

9. Gagnadoux MF, Habib R, Gubler MC, Bacri JL, Broyer M. Long term (15-25 years) outcome of childhood hemolytic uremic syndrome. Clin Nphrol. 1996;46:39-41.

10. Scully M, McDonald V, Cavenagh J, et al. A phase II study of the safety and efficacy of rituximab with plasma exchange in acute acquired thrombotic thrombocytopenic purpura. Blood. 2011;118(7):1746-1753.

11. Wiegand TJ, Tarabar A. Nonsteroidal Anti-inflammatory Agent Toxicity. http://emedicine.medscape.com/article/816117-medication. Accessed Nov 14, 2012.

12. Parente L, Perretti M. Advances in the pathophysiology of constitutive and inducible cyclooxygenases: two enzymes in the spotlight. Biochem Pharmacol. 2003;65(2):153-159.

13. Court H, Volans GN. Poisoning after overdose with non-steroidal antiinflammatory drugs. Adverse Drug React Acute Poisoning Rev. 1984;3(1):1-21.

14. Virji MA, Venkataraman ST, Lower DR, Rao KN. Role of laboratory in the management of phenylbutazone poisoning. J Toxicol Clin Toxicol. 2003;41(7): 1013-1024.

15. Furlan M, Lämmle B. Aetiology and pathogenesis of thrombotic thrombocytopenic purpura and haemolyticuraemic syndrome: the role of von Willebrand factor-cleaving protease. Best Pract Res Clin Haematol. 2001;14(2):437-454.

16. Pharmacia \& Upjohn Company. Motrin ${ }^{\circledR}$ (ibuprofen tablets USP) prescribing information. Kalamazoo, MI; 2000.

17. Bronstein AC, Spyker DA, Cantilena LR, Green JL, Rumack BH, Dart RC. 2010 Annual Report of the American Association of Poison Control Centers' National Poison Data System (NPDS): 28th Annual Report. Clin Toxicol (Pbila). 2011;49(10):910-941.

18. Schoenmaker NJ, WeeningJJ, Krediet RT. Ibuprofen-induced HUS. Clin Nephrol. 2007;68(3):177-178.

19. Lian EC, Savaraj N. Effects of platelet inhibitors on the platelet aggregation induced by plasma from patients with thrombotic thrombocytopenic purpura. Blood. 1981;58(2):354-359.

20. Hensby CN, Lewis PJ, Hilgard P, Mufti GJ, Hows J, Webster J. Prostacyclin deficiency in thrombotic thrombocytopenic purpura. Lancet. 1979;2(8145):748. 